\title{
Subthalamic Nucleus Deep Brain Stimulation May Reduce Medication Costs in Early Stage Parkinson's Disease
}

\author{
Mallory L. Hacker ${ }^{\mathrm{a}}$, Amanda D. Currie ${ }^{\mathrm{a}}$, Anna L. Molinari ${ }^{\mathrm{a}}$, Maxim Turchan ${ }^{\mathrm{a}}$, Sarah M. Millan ${ }^{\mathrm{a}}$, \\ Lauren E. Heusinkveld ${ }^{\mathrm{a}}$, Jonathon Roach ${ }^{\mathrm{a}}$, Peter E. Konrad ${ }^{\mathrm{b}}$, Thomas L. Davis ${ }^{\mathrm{a}}$, Joseph S. Neimat ${ }^{\mathrm{b}}$, \\ Fenna T. Phibbs ${ }^{\mathrm{a}}$, Peter Hedera ${ }^{\mathrm{a}}$, Daniel W. Byrne ${ }^{\mathrm{c}}$ and David Charles ${ }^{\mathrm{a}, *}$ \\ ${ }^{a}$ Department of Neurology, Vanderbilt University, Medical Center North, Nashville, TN, USA \\ ${ }^{\mathrm{b}}$ Department of Neurosurgery, Vanderbilt University, Village at Vanderbilt, Nashville, TN, USA \\ ${ }^{\mathrm{c}}$ Department of Biostatistics, Vanderbilt University, West End, Suite Nashville, TN USA
}

Accepted 4 January 2016

\begin{abstract}
.
Background: Subthalamic nucleus deep brain stimulation (STN-DBS) is well-known to reduce medication burden in advanced stage Parkinson's disease (PD). Preliminary data from a prospective, single blind, controlled pilot trial demonstrated that early stage PD subjects treated with STN-DBS also required less medication than those treated with optimal drug therapy (ODT).

Objective: The purpose of this study was to analyze medication cost and utilization from the pilot trial of DBS in early stage PD and to project 10 year medication costs.

Methods: Medication data collected at each visit were used to calculate medication costs. Medications were converted to levodopa equivalent daily dose, categorized by medication class, and compared. Medication costs were projected to advanced stage PD, the time when a typical patient may be offered DBS.

Results: Medication costs increased 72\% in the ODT group and decreased 16\% in the DBS+ODT group from baseline to 24 months. This cost difference translates into a cumulative savings for the DBS+ODT group of $\$ 7,150$ over the study period. Projected medication cost savings over 10 years reach $\$ 64,590$. Additionally, DBS+ODT subjects were $80 \%$ less likely to require polypharmacy compared with ODT subjects at 24 months ( $p<0.05$; OR $=0.2 ; 95 \%$ CI: $0.04-0.97)$.

Conclusions: STN-DBS in early PD reduced medication cost over the two-year study period. DBS may offer substantial long-term reduction in medication cost by maintaining a simplified, low dose medication regimen. Further study is needed to confirm these findings, and the FDA has approved a pivotal, multicenter clinical trial evaluating STN-DBS in early PD.
\end{abstract}

Keywords: Parkinson's disease, deep brain stimulation, subthalamic nucleus, medication cost, cost, cost analysis, health economics

\footnotetext{
${ }^{*}$ Correspondence to: David Charles, MD, Department of Neurology, Vanderbilt University, 161121 st Ave S. A-1106 Medical Center North Nashville, TN 37232-2551, USA. Tel.: +1 615322 2538; Fax: +1 615322 0262; E-mail: david.charles@vanderbilt.edu.
}

\section{INTRODUCTION}

Subthalamic nucleus deep brain stimulation (STNDBS) is an effective adjunctive therapy for advanced stage Parkinson's disease (PD) that improves motor symptoms and quality of life while also 
reducing medication requirements and complications associated with medical therapy [1]. A prospective, randomized, single blind, pilot clinical trial demonstrated initial safety and tolerability of STN-DBS in early stage PD [2], and the FDA has approved a pivotal, phase III, multicenter, safety and efficacy trial evaluating DBS in early PD. In the pilot trial, early PD subjects randomized to receive DBS plus optimal drug therapy (DBS+ODT) required less medications than the ODT group at each follow-up visit [2], which is consistent with the established medication reduction associated with STN-DBS in advanced stage PD. Medication costs are one of the most important economic drivers of healthcare expenditures, and increasing cost negatively impacts patient access to and compliance with prescribed therapies. Here, we report a medication cost analysis from the only prospective study of DBS in early stage PD and offer a projection of medication cost from early PD until the time that a typical patient would be treated with DBS [1].

\section{MATERIALS AND METHODS}

Investigators at Vanderbilt University conducted a prospective, randomized, single-blind clinical trial testing STN-DBS in early stage PD (NCT\#00282152) from 2006-2012 that was approved by the FDA (IDE\#G050016) and Vanderbilt University IRB (IRB\#040797). Study design, enrollment experience, baseline characteristics, and outcomes are published elsewhere [2-5]. Briefly, the pilot enrolled thirty idiopathic PD subjects, age 50-75 years (60.2 \pm 6.6$)$, Hoehn \& Yahr Stage II off medication, with a medication duration of 6 months to 4 years $(2.1 \pm 1.5)$ without dyskinesias or other motor fluctuations who provided written informed consent. After baseline evaluations, subjects were randomized to receive ODT or DBS+ODT. Subjects' PD medication regimens were recorded at each visit (baseline, 6, 12, 18, and 24 months) and converted to levodopa equivalents (LEDD) [6]. This analysis includes all subjects who completed at least one follow-up visit $(n=29)$.

To minimize bias, the subject's treating neurologist (not the principal investigator) was responsible for medication adjustments and could use any available therapy to manage PD symptoms. The following PD medications and classes were identified in this cohort: Levodopa - carbidopa/levodopa; Levodopa+COMT Inhibitor - carbidopa/levodopa/ entacapone; Dopamine Agonists - pramipexole, rotigotine, ropinirole; MAO-B Inhibitors - selegeline, rasagiline; Antiviral - amantadine [7]. Medication strengths, doses, and frequencies were collected at each visit and verified by medical record chart review.

Daily medication costs were calculated using prices listed in the Drug Topics Red Book (2010 edition). The lowest cost available was selected for medications with multiple pill quantities listed. Generic costs were used if a generic medication was available at the time of study visit. Medication data collected at each visit in the pilot were used to calculate the average daily cost for PD medications. Cumulative annual costs during the pilot were calculated by adding annual costs from 12 months and 24 months.

For cost projections, a $10 \%$ annual increase in medication cost was projected for ODT and DBS+ODT groups due to disease progression and greater complexity of PD medications [8]. Additionally, a reduced annual increase in medication cost $(5 \%)$ was also projected for the DBS+ODT group because subjects receiving STN-DBS at any stage of illness often utilize significantly less medication $[1,9]$.

Baseline daily medication costs were compared using a Mann-Whitney $U$ test. To account for the small sample size, a likelihood ratio test was used to compare polypharmacy status between the two groups.

\section{RESULTS}

Average baseline daily costs were similar for the ODT and DBS+ODT groups, \$13.81 $( \pm \$ 6.98)$ and $\$ 13.39$ ( $\pm \$ 5.98)$, respectively ( $p=0.70$; Fig. 1A). Daily cost increased for the ODT group at each sixmonth visit, reaching a $72 \%$ increase from baseline by 24 months ( $\$ 23.82 \pm \$ 27.95)$. Conversely, after STN-DBS surgery, the DBS+ODT group medication cost was less than baseline values at each followup visit, with a $16 \%$ decrease from baseline at 24 months $(\$ 11.31 \pm \$ 7.80)$. Annual medication costs at 24 months were $\$ 8,694$ for the ODT group and $\$ 4,128$ for the DBS+ODT group. The average cumulative medication costs from the pilot were $\$ 14,662$ and $\$ 7,512$ for the ODT and DBS+ODT groups, respectively (Fig. 1A). This between-group difference during the two-year pilot translates into a total cumulative medication cost savings of $\$ 7,150$ for the DBS+ODT group. 

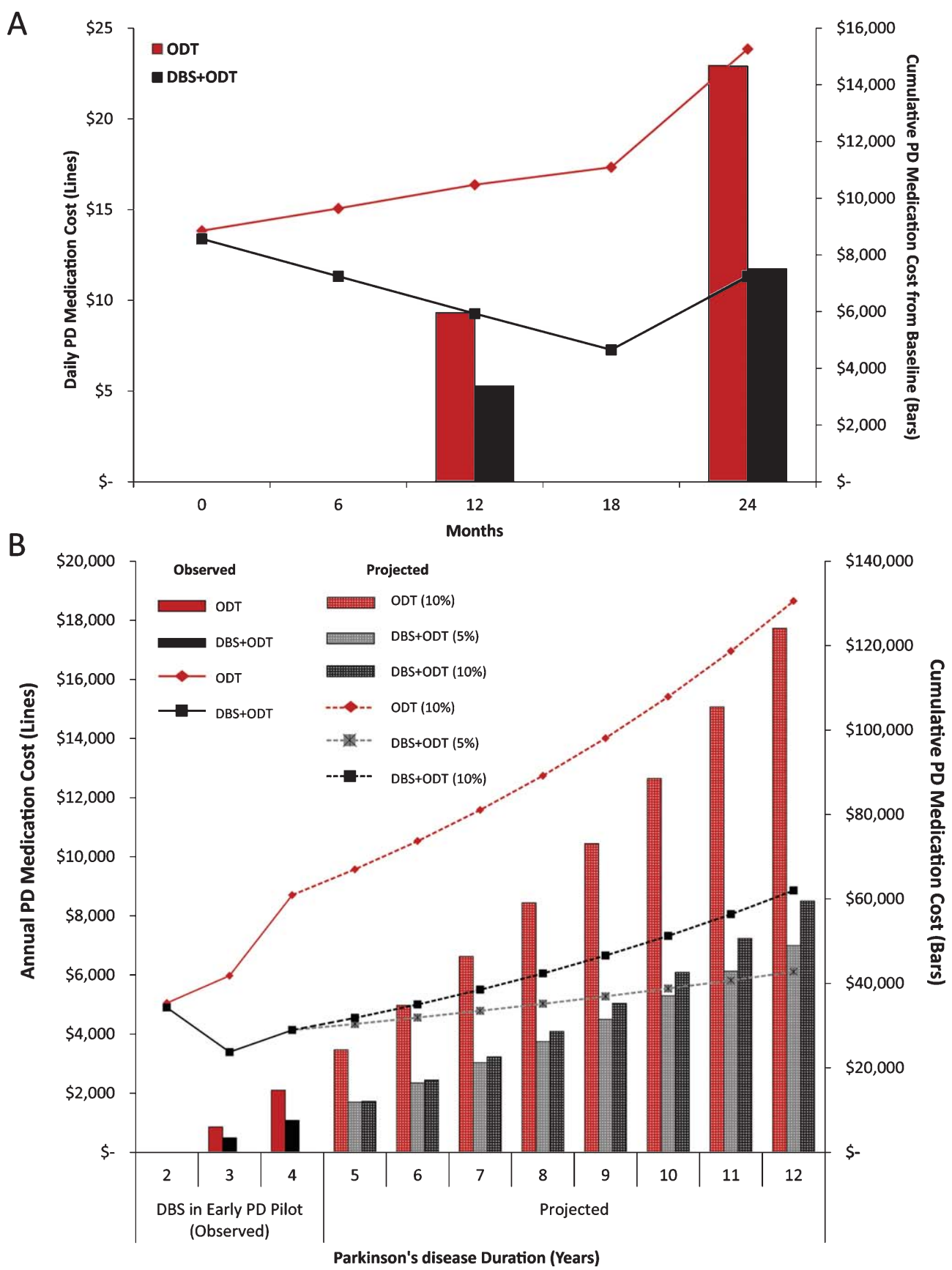

Fig. 1. Medication cost and projections from the DBS in early PD pilot trial. (A) Average daily medication costs at each visit (lines, left y-axis). Average total cumulative annual medication costs from baseline at 12 months and 24 months (bars, right y-axis). (B) Annual (left $y$-axis) and cumulative (right y-axis) medication costs observed for ODT and DBS+ODT groups based on the pilot (cumulative medication costs are shown from the DBS in early PD pilot trial baseline). Annual medication increases of $10 \%$ were projected for ODT and DBS+ODT groups, and an increase of 5\% is also projected for the DBS+ODT group.

Figure 1B presents annual and cumulative medication costs projected from the conclusion of the pilot trial in early stage PD (average disease duration $=2.1$ years [2]) through advanced stage disease. A $10 \%$ annual increase in medication cost was assigned to both groups due to disease progression and greater complexity of PD medications [8]. Additionally, a reduced annual increase in medication cost $(5 \%)$ 
is also projected for the DBS+ODT group based on the established medication reduction associated with STN-DBS in advanced PD stages [1]. Based on $10 \%$ increase estimates, annual medication costs will reach $\$ 18,637$ for the ODT group and $\$ 8,849$ for the DBS+ODT group in advanced PD (12 years disease duration), and DBS+ODT is projected to reduce the total cumulative medication costs by $\$ 64,590$ over 10 years. Furthermore, medication cost savings projected using a reduced $5 \%$ annual medication cost increase for the DBS+ODT group would reach $\$ 75,129$ after 10 years.

Because medication costs are directly influenced by the classes of medications prescribed, we analyzed medication classes contributing to total LEDD. Total LEDD for the ODT group was increased from baseline at each follow-up visit, with a $50 \%$ increase by 24 months (Fig. 2A, [2]). This increase in total LEDD for the ODT group is largely driven by increased utilization of the levodopa+COMT inhibitor medication class (Fig. 2B). Total LEDD decreased in the DBS+ODT group at each visit from baseline through 18 months and then rose to a $16 \%$ increase by 24 months (Fig. 2A). When medication need did increase in the DBS+ODT group, levodopa was most commonly utilized (Fig. 2B). Furthermore, dopamine agonists for the DBS+ODT group were reduced from baseline at each visit, reaching a $25 \%$ reduction by 24 months. Finally, the proportion of ODT subjects on polypharmacy increased from $43 \%$ to $71 \%$ (baseline to 24 months), while there was no increase in the proportion of DBS+ODT subjects on polypharmacy $(33 \%)$ at the end of the pilot trial (Fig. 2C). ODT subjects were 5 times more likely to require polypharmacy at 24 months compared to DBS+ODT subjects $(p<0.05 ; \mathrm{OR}=5.0 ; 95 \% \mathrm{CI}$ 1.03-24.28).

\section{DISCUSSION}

This study reports PD medication cost savings of $\$ 7,150$ over two years with STN-DBS in early stage PD. It is well established that STN-DBS significantly reduces medication utilization in advanced PD. Therefore, projections were projections were calculated for medication cost over the time from early PD until a typical advanced stage patient would be offered DBS. Using conservative assumptions, substantial cumulative medication cost savings are predicted to result when STN-DBS is applied in early PD (\$64,590 over 10 years).
The costs associated with DBS (such as the device, surgical procedures, and battery replacements) make it a significantly more expensive treatment option than standard medical therapy; however, this additional cost is justified for advanced PD patients by the superior quality of life, improved motor function, and decreased complications of medical therapy that DBS provides [1]. Although the savings projected here do not outweigh the costs associated with STNDBS, the predicted long-term medication reduction and associated decrease in medication costs represent a significant offset. PD progression requires higher doses of medications used in more complex combinations, which increases total costs. Indeed, the medication cost savings observed for the DBS+ODT group in this study (Fig. 1A) is attributed to not only reduced total medication requirement (Fig. 2A-B) but also the ability to maintain a simpler medication regimen compared to the ODT group (Fig. 2C). Additionally, because STN-DBS in advanced PD reduces medication utilization, the associated complications and side effects are also likely reduced [1]. Application of DBS in early stage PD, prior to the onset of these medication associated complications and side effects, has the potential to suppress their development by simply imparting less medication need over the course of the disease.

There are a number of limitations that should be considered for this analysis in addition to surgical risks associated with this line of research. Medications were not adjusted using a standardized treatment algorithm, which is a limitation of this study. To reduce bias however, medications in the pilot were managed by the subject's treating neurologist (not the principal investigator) who could use any available therapies to manage PD symptoms. The pilot trial was an open-label, single-blind study, and therefore placebo or lessebo effects may have influenced subjects' medication utilization [10]. The study is limited by its small sample size, and this analysis only reflects the PD medications that were available during the study period (2006-2012). While generic versions are now available for some of the medications utilized during this trial, it is important to note that the landscape of PD medications continues to evolve. New pharmacological options are associated with significant cost, and therefore, the projections presented here likely underestimate future savings. There are surgical risks associated with DBS therapy when it is applied in early, mid, or late stage disease. Additional study is needed to determine if these risks are outweighed by the 

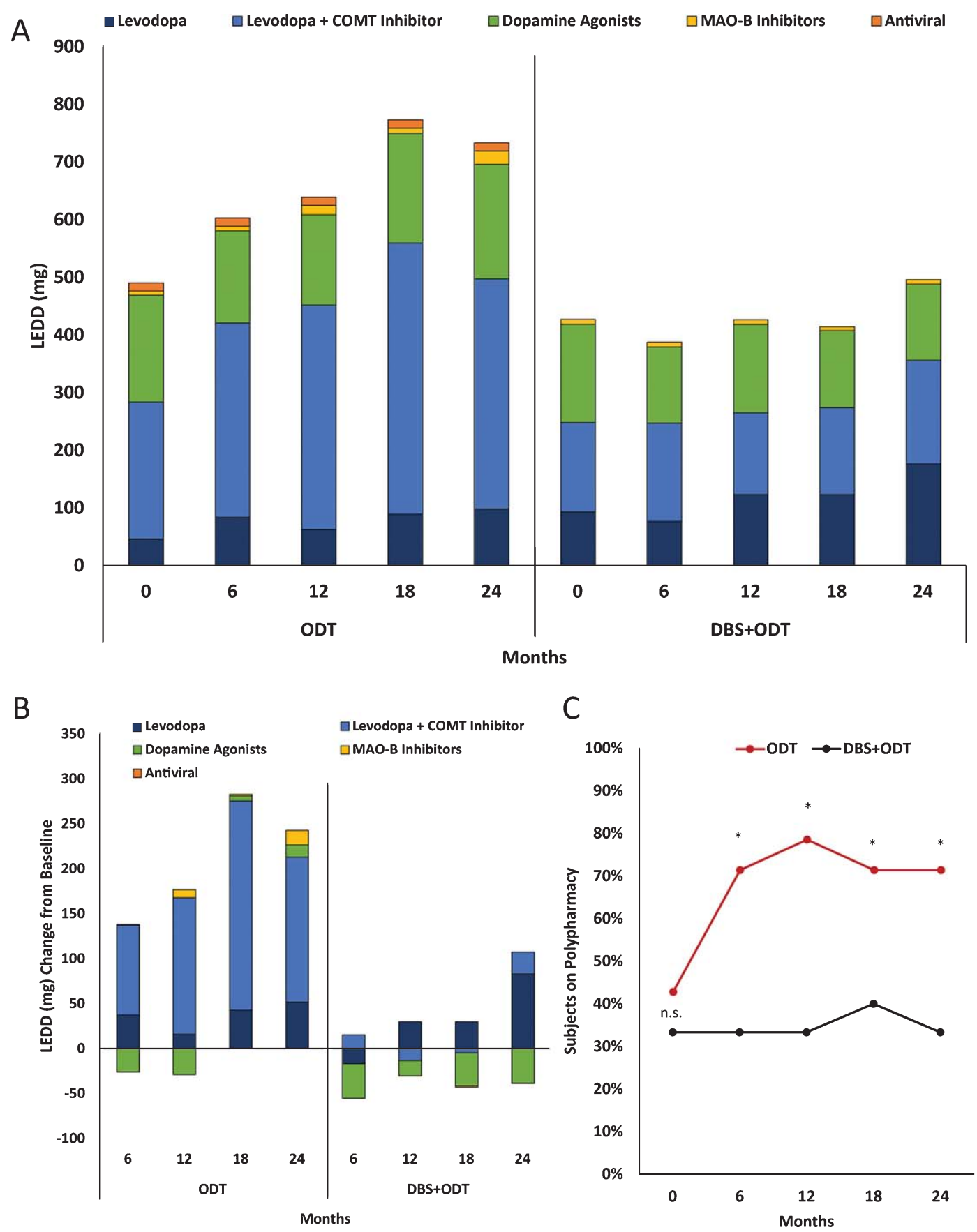

Fig. 2. Medication class contributions. (A) Average relative contributions of medication classes to total LEDD (mg) over the two-year study period. (B) Average change from baseline for LEDD (mg) by medication class. (C) Proportion of subjects prescribed more than one type of PD medication (polypharmacy) at baseline and each six-month visit. n.s. $=$ not significant, ${ }^{*}=p<0.05$.

benefit of the therapy if applied in early stage disease. Potential research participants are entitled to make informed decisions regarding their clinical care and their participation in studies of DBS in early stage PD [11]. Without a biomarker for Parkinson's disease, there is a chance that early application of DBS will include patients who are later determined to have a condition other than idiopathic PD. The Movement Disorders Society has recently published new guidelines that could be helpful in reducing 
this occurrence [12], and it is important to note that there were no subjects in the pilot trial who have had an alternate diagnosis after 5 years of follow-up $(n=29)$.

A conservative approach was taken for this analysis and projection to avoid exaggerating cost savings. In 2004, we reported annual medication costs for advanced PD subjects prior to receiving STN-DBS to be $\$ 7,172$ [8], and that report offered estimates of the impact of inflation on medication costs over time. Advanced PD patients without STN-DBS from that 2004 study would have annual medication costs reach $\$ 20,462$ in 2015 prices (using a $10 \%$ annual medication cost inflation rate) [8], which closely aligns with our current projection of annual cost for advanced stage PD treated with ODT $(\$ 18,637$; Fig. 1B). Additionally, two projections of annual increase in medication cost for the DBS+ODT group are presented. A $10 \%$ annual medication increase rate is presented for both ODT and DBS+ODT groups (10 year medication cost savings projection $=\$ 64,590$ ), and a reduced annual medication increase of $5 \%$ is also projected to reflect the well-established ability of STN-DBS to decrease PD medication burden and cost (10 year medication cost savings projection $=\$ 75,129)$. Moreover, inflation was not factored into the analysis presented here. If more aggressive assumptions are applied, such as a $10 \%$ annual increase due to medication cost inflation, the projected medication savings resulting from STN-DBS in early stage PD would reach $\$ 81,927$.

The long-term PD medication cost savings of STN-DBS in early stage PD are secondary to the need for improved patient outcomes such as better motor function and quality of life and suppression of the complications of medical therapy. DBS+ODT is proven to offer superior outcomes to ODT in all PD stages tested thus far [1,9]. A multicenter, randomized controlled trial of STN-DBS in mid-stage PD demonstrated significant medication reduction with DBS+ODT after two years which is predicted to result in significant medication cost savings [9]. While the cost of DBS therapy is significant, STN-DBS applied in early stage PD may impart a substantial reduction in medication utilization and cost over many more years than when DBS is applied in current standard of care (Fig. 1). Even though these savings do not exceed the cost of DBS therapy, they do represent a substantial offset when considered over the course of the disease. Additional investigation into the safety and efficacy of DBS in early stage PD is indicated, and the FDA has approved a piv- otal, multicenter, phase III, clinical trial evaluating STN-DBS in early stage PD.

\section{ACKNOWLEDGMENTS}

Research reported in this publication was supported by Medtronic, Inc., Vanderbilt CTSA grant UL1TR000445 from the National Center for Advancing Translational Sciences (NCATS), NCATS/NIH award UL1TR000011, NIH R01 EB006136, private donations and The Michael J. Fox Foundation for Parkinson's Research. Medtronic representatives did not take part in data collection, management, analysis, or interpretation or in preparation, review, or approval of the manuscript.

\section{CONFLICT OF INTEREST}

Vanderbilt University receives income in excess of $\$ 10,000$ from grants or contracts from Allergan, Ipsen, Merz, and Medtronic for research or educational programs led by David Charles. David Charles receives income from Allergan, Ipsen, Merz, and Medtronic for education or consulting services. Peter E. Konrad receives research funding and is on the speaker's bureau for Medtronic. Fenna T. Phibbs has done consulting work for Medtronic, Boston Scientific, and St. Jude Medical. Joseph S. Neimat has done consulting work for Medtronic and has received research funding from Medtronic. There are no conflicts of interest for Mallory L. Hacker, Amanda D. Currie, Anna L. Molinari, Maxim Turchan, Sarah M. Millan, Lauren E. Heusinkveld, Jonathon Roach, Thomas L. Davis, Peter Hedera, or Daniel W. Byrne.

\section{REFERENCES}

[1] Deuschl G, Schade-Brittinger C, Krack P, Volkmann J, Schäfer H, Bötzel K, Daniels C, Deutschländer A, Dillmann U, Eisner W, Gruber D, Hamel W, Herzog J, Hilker R, Klebe S, Kloss M, Koy J, Krause M, Kupsch A, Lorenz D, Lorenzl S, Mehdorn HM, Moringlane JR, Oertel W, Pinsker MO, Reichmann H, Reuß A, Schneider G-H, Schnitzler A, Steude U, Sturm V, Timmermann L, Tronnier V, Trottenberg T, Wojtecki L, Wolf E, Poewe W, \& Voges J (2006) A randomized trial of deep-brain stimulation for Parkinson's disease. $N$ Engl J Med, 355, 896-908.

[2] Charles D, Konrad PE, Neimat JS, Molinari AL, Tramontana MG, Finder SG, Gill CE, Bliton MJ, Kao C, Phibbs FT, Hedera P, Salomon RM, Cannard KR, Wang L, Song Y, \& Davis TL (2014) Subthalamic nucleus deep brain stimulation in early stage Parkinson's disease. Parkinsonism Relat Disord, 20, 731-737.

[3] Charles D, Tolleson C, Davis TL, Gill CE, Molinari AL, Bliton MJ, Tramontana MG, Salomon RM, Kao C, Wang 
L, Hedera P, Phibbs FT, Neimat JS, \& Konrad PE (2012) Pilot study assessing the feasibility of applying bilateral subthalamic nucleus deep brain stimulation in very early stage Parkinson's disease: Study design and rationale. J Parkinsons Dis, 2, 215-223.

[4] Hacker ML, Tonascia J, Turchan M, Currie A, Heusinkveld L, Konrad PE, Davis TL, Neimat JS, Phibbs FT, Hedera P, Wang L, Shi Y, Shade DM, Sternberg AL, Drye LT, \& Charles D (2015) Deep brain stimulation may reduce the relative risk of clinically important worsening in early stage Parkinson's disease. Parkinsonism Relat Disord, 21, 11771183.

[5] Charles PD, Dolhun RM, Gill CE, Davis TL, Bliton MJ, Tramontana MG, Salomon RM, Wang L, Hedera P, Phibbs FT, Neimat JS, \& Konrad PE (2012) Deep brain stimulation in early Parkinson's disease: Enrollment experience from a pilot trial. Parkinsonism Relat Disord, 18, 268-273.

[6] Tomlinson CL, Stowe R, Patel S, Rick C, Gray R, \& Clarke CE (2010) Systematic review of levodopa dose equivalency reporting in Parkinson's disease. Mov Disord, 25, 26492653.

[7] Weaver FM, Stroupe KT, Cao L, Holloway RG, Vickrey BG, Simuni T, Hendricks A, \& Ippolito D (2012) Parkinson's disease medication use and costs following deep brain stimulation. Mov Disord, 27, 1398-1403.

[8] Charles PD, Padaliya BB, Newman WJ, Gill CE, Covington CD, Fang JY, So SA, Tramontana MG, Konrad PE, \& Davis TL (2004) Deep brain stimulation of the subthalamic nucleus reduces antiparkinsonian medication costs. Parkinsonism Relat Disord, 10, 475-479.
[9] Schuepbach WMM, Rau J, Knudsen K, Volkmann J, Krack P, Timmermann L, Hälbig TD, Hesekamp H, Navarro SM, Meier N, Falk D, Mehdorn M, Paschen S, Maarouf M, Barbe MT, Fink GR, Kupsch A, Gruber D, Schneider GH, Seigneuret E, Kistner A, Chaynes P, Ory-Magne F, Courbon CB, Vesper J, Schnitzler A, Wojtecki L, Houeto JL, Bataille B, Maltête D, Damier P, Raoul S, Sixel-Doering F, Hellwig $\mathrm{D}$, Gharabaghi A, Krüger R, Pinsker MO, Amtage F, Régis JM, Witjas T, Thobois S, Mertens P, Kloss M, Hartmann A, Oertel WH, Post B, Speelman H, Agid Y, Schade-Brittinger C, \& Deuschl G (2013) Neurostimulation for Parkinson's disease with early motor complications. $N$ Engl J Med, 368, 610-622.

[10] Mestre TA, Espay AJ, Marras C, Eckman MH, Pollak P, \& Lang AE (2014) Subthalamic nucleus-deep brain stimulation for early motor complications in Parkinson's disease: The EARLYSTIM Trial: Early is not always better. Mov Disord, 29, 1751-1756.

[11] Finder SG, Bliton MJ, Gill CE, Davis TL, Konrad PE, \& Charles PD (2012) Potential subjects" responses to an ethics questionnaire in a phase I study of deep brain stimulation in early Parkinson"s disease. J Clin Ethics, 23, 207-216.

[12] Postuma RB, Berg D, Stern M, Poewe W, Olanow CW, Oertel W, Obeso J, Marek K, Litvan I, Lang AE, Halliday G, Goetz CG, Gasser T, Dubois B, Chan P, Bloem BR, Adler $\mathrm{CH}, \&$ Deuschl G (2015) MDS clinical diagnostic criteria for Parkinson's disease. Mov Disord, 30, 1591-1601. 